\title{
MicroRNA-30e protects the heart against ischemia and reperfusion injury through autophagy and the Notch1/Hes1/Akt signaling pathway
}

\author{
JIANJIE ZHENG, JING LI, BO KOU, QIUYUE YI and TAO SHI \\ Department of Cardiovascular Surgery, The First Affiliated Hospital of Medical College of Xi'an Jiaotong University, \\ Xi'an, Shaanxi 710061, P.R. China
}

Received January 24, 2017; Accepted February 28, 2018

DOI: $10.3892 / \mathrm{ijmm} .2018 .3548$

\begin{abstract}
The aim of the present study was to determine the cardioprotective mechanisms by which micro (mi)RNA-30e protects the heart from myocardial ischemia/reperfusion injury (MI/R) and to explore the signaling pathways that may confer protection for the heart and be potential therapeutic targets. It was demonstrated that miRNA-30e expression was decreased in patients with MI/R. In H9C2 cells, silencing (si)miRNA-30e significantly inhibited cellular apoptosis, the expression of apoptosis regulator BAX (Bax) and caspase-3 activity. It also significantly increased the expression of microtubule-associated proteins 1A/1B light chain 3B, p62, Beclin-1, neurogenic locus notch homolog protein-1 (Notch1), Hes1 and phosphorylated-protein kinase B (p-Akt), and decreased the expression of inducible NO synthase (iNOS) and proteins associated with oxidative stress. The inhibition of autophagy following treatment with 3-methyladenine significantly reversed the effect of si-miRNA-30e on apoptosis, Bax, caspase-3, iNOS and oxidative stress in $\mathrm{H} 9 \mathrm{C} 2$ cells. The promotion of Notch1 expression increased the effect of si-miRNA-30e on apoptosis, Bax, caspase-3, iNOS, Notch1, Hes1 and p-Akt protein expression and oxidative stress in $\mathrm{H} 9 \mathrm{C} 2$ cells. Taken together, these results indicate that miRNA-30e protects the heart from MI/R via autophagy and the Notch1/Hes1/Akt signaling pathway.
\end{abstract}

\section{Introduction}

Myocardial ischemia/reperfusion injury (MI/R), which occurs following myocardial infarction, is caused by the sudden,

Correspondence to: Dr Jianjie Zheng, Department of Cardiovascular Surgery, The First Affiliated Hospital of Medical College of Xi'an Jiaotong University, 277 Yanta West, Xi'an, Shaanxi 710061, P.R. China

E-mail: yzjxvdq20041@126.com

Key words: microRNA-30e, heart ischemia, reperfusion injury, autophagy, neurogenic locus notch homolog protein 1, transcription factor HES-1, protein kinase B complete interruption of the myocardial blood circulation, which leads to myocardial necrosis (1). MI/R is a major health problem in China and its morbidity and mortality rates increase every year (1). The development of acute heart failure is the primary cause of mortality following acute MI/R (2). The advancement of medical techniques, such as internal medicine thrombolysis, interventional stent and bypass surgery, have markedly reduced the mortality rate of patients with acute $\mathrm{MI} / \mathrm{R}$ that consequently experience acute heart failure (3). However, MI/R may develop into a chronic disease as it progresses; the chronic stage of the disease is dominated by myocardial fibrosis remodeling, which may induce chronic heart failure and lead to mortality (4).

Micro (mi)RNA acts on target genes and regulates expression of these genes, influencing the relevant molecular signaling pathways of progenitor cell activation, thus regulating multiple biological and pathological processes (5). MiRNA also serves an important role in pathological processes, including myocardial ischemia and ventricular remodeling following ischemia and infarction (6). Furthermore, miRNA serves an important role in cardiac development and myogenic differentiation, and protects against myocardial ischemia (6). The neurogenic locus notch homolog protein (Notch) signaling pathway serves a role in regulating cardiac development, as well as the proliferation and differentiation of myocardial cells (7). Therefore, miRNA may regulate cardiac development, as well as the proliferation and differentiation of myocardial cells by activating the Notch signaling pathway.

Autophagy occurs in normal cardiac myocardium, however, it is increased in hearts affected by myocardial hypertrophy and ischemia (8). Autophagy has been demonstrated to support the adaptive response of the heart, thus protecting the heart from hemodynamic overload and acute ischemic death (9). However, cell death induced by autophagy also occurs following MI/R (9). It is therefore important to identify the impact of myocardial autophagy and the mechanisms by which it occurs in order to to develop novel therapies to prevent and treat post-MI/R myocardial remodeling (10).

A number of signaling pathways are involved in the polarization of inflammation of MI/R (11). The Notch signaling pathway has been conserved through evolution and serves an important role in regulating cell growth, development, 
differentiation and apoptosis, as well as the post-injury remodeling of tissue (11). The results of a study into the cardiac development of fruit flies demonstrated that the Notch signaling pathway primarily regulates cardiac development in two ways: Asymmetric differentiation and lateral inhibition (12). The Notch signaling pathway induces the asymmetric division of heart progenitor cells via asymmetric differentiation and the expression of the Notch signal differs between the two daughter cells; therefore it induces different effects in the two daughter cells (13). It has been identified in a previous study on the cardiac development of mice that receptors of the Notch signaling pathway were primarily expressed during different stages of development: Notch1 and Notch2 were highly expressed during cardiac development, whereas Notch3 exhibited high expression in smooth muscle and Notch4 was highly expressed in the vascular endothelium (14).

The downstream molecules of the Notch signaling pathway possess the characteristic structure of a basic helix-loop-helix and belong to the Hes and Hey families (15). In mammals, the Hes family is comprised of seven genes (Hes1-7), of which only Hes1, Hes5 and Hes7 are regulated by the Notch signaling pathway (15). From the Hey family, Hey1, Hey2 and Hey L are regulated by the Notch signaling pathway (15). A mouse embryo with a mutation in the Notch1 gene exhibits cardiac defects, suggesting that the Notch signaling pathway is essential for the early morphology and remodeling of the heart in vertebrates (16). During early cardiac development, the expression of Hes1 is restricted to the atria; by contrast, Hes2 is expressed in the ventricles (17). Following birth, the expression of Hes1 and Hes2 in the heart gradually decrease (17).

The protein kinase $\mathrm{B}(\mathrm{Akt})$ signaling pathway is involved in cytophysiological processes, for example, it regulates the mitochondrion-mediated anti-apoptosis process (16). The results of a recent study demonstrated that the Akt signaling cascade was involved in the production of antioxidant enzymes in the mitochondria and suggested that the Akt signaling cascade may be an important upstream regulatory mechanism that increases the activity of antioxidant enzymes in the cytoplasm (18). Lai et al (19) demonstrated that miRNA-30e induces cardioprotection in rats with doxorubicin-induced heart failure via autophagy. Therefore, the present study examined the cardioprotective mechanisms by which miRNA-30e protects the heart against MI/R. The specific signaling pathways that may provide protection and be a potential target for novel therapies to treat patients with heart disease were also explored.

\section{Materials and methods}

Patients. Peripheral blood samples were collected from 24 patients (all male, aged 47-63 years old) with MI/R admitted to the Department of Cardiac Surgery at The First Affiliated Hospital of Medical College of Xi'an Jiaotong University, as well as 24 age- and sex-matched healthy volunteers (all male, aged 45-65 years old) between August and September 2016. Peripheral blood was centrifuged at 2,000 x g for $10 \mathrm{~min}$ at $4^{\circ} \mathrm{C}$; subsequently, serum was collected and stored at $-80^{\circ} \mathrm{C}$. The present study was approved by the Ethics Committee of the First Affiliated Hospital of Xi'an Jiaotong University (Xi'an, China). All patients provided written informed consent prior to the collection of blood samples.
Reverse transcription-quantitative polymerase chain reaction $(R T-q P C R)$ analysis. Total RNA was extracted from collected serum or rat myocardium H9C2 cells (Shanghai Cell Bank of Chinese Academy of Sciences, Shanghai, China) using TRIzol Reagent (Invitrogen; Thermo Fisher Scientific, Inc., Waltham, MA, USA). Subsequently, $1 \mu \mathrm{g}$ total RNA was reverse-transcribed into cDNA using the RealMasterMix First Strand cDNA Synthesis kit (Tiangen Biotech Co., Ltd., Beijing, China) following the manufacturer's protocol. The relative expression of miRNA-30e was mixed. qPCR was performed using SYBR ${ }^{\circledR}$ Premix ExTaq ${ }^{\mathrm{TM}}$ (Takara Bio, Inc., Otsu, Japan) on an Applied Biosystems 7500 real-time PCR system (Thermo Fisher Scientific, Inc.). The qPCR conditions were as follows: $95^{\circ} \mathrm{C}$ for $10 \mathrm{~min}, 40$ cycles of $95^{\circ} \mathrm{C}$ for $30 \mathrm{sec}$ and $60^{\circ} \mathrm{C}$ for $30 \mathrm{sec}$. The following primers were used: miRNA-30e, forward, 5'-GGC GGTGTAAACATCCTT-3' and reverse, 5'-GTCGTATCC AGTGCAGGGTCCGAGGTATTCG-3'; U6, forward, 5'-CTCGCTTCGGCAGCACA-3' and reverse, 5'-AACGCT TCACGAATTTGCGT-3'. miRNA-30e expression was quantified using the $2^{-\Delta \Delta \mathrm{Cq}}$ method (20).

Cell culture and transfection. Cardiac myoblast $\mathrm{H} 9 \mathrm{C} 2$ cells were cultured in Dulbecco's modified Eagle's medium (DMEM, Gibco; Thermo Fisher Scientific, Inc.) supplemented with $10 \%$ fetal bovine serum (Gibco; Thermo Fisher Scientific, Inc.) and $100 \mathrm{U}$ penicillin and streptomycin at $37^{\circ} \mathrm{C}$ in a humidified atmosphere containing $5 \% \mathrm{CO}_{2}$. si-miRNA-30e and negative mimics were purchased from Sangon Biotech Co., Ltd. (Shanghai, China). A total of $200 \mathrm{ng}$ si-miRNA-30e (5'-AAAGAGCAGTGTTTCGT-3' and 5'-CCGACC GTCGGTAAA-3') and $200 \mathrm{ng}$ negative control (5'-CCC CCCCCCCC-3' and 5'-CCCCCCCCC-3') mimics were co-transfected into H9C2 cells using Lipofectamine ${ }^{\circledR} 2000$ (Invitrogen; Thermo Fisher Scientific, Inc.). Following $6 \mathrm{~h}$ transfection, cells were incubated in a hypoxic chamber $(5 \%$ $\mathrm{CO}_{2}$ and $95 \%$ nitrogen) at $37^{\circ} \mathrm{C}$ for $2 \mathrm{~h}$ to establish an in vitro model of MI/R, following a previously published protocol (21).

Treatment with 3-methyladenine (3-MA). Following $6 \mathrm{~h}$ transfection, cells were incubated with $10 \mu \mathrm{M} 3-\mathrm{MA}$ (MedChemExpress, Monmouth Junction, NJ, USA) in a hypoxic chamber $\left(5 \% \mathrm{CO}_{2}\right.$ and $95 \%$ nitrogen) at $37^{\circ} \mathrm{C}$ for $2 \mathrm{~h}$.

Notch1 treatment. Notch1 recombinant protein was purchased from Beijing Yiqiao Shenzhou Technology Co. Ltd. (Beijing, China). Following $6 \mathrm{~h}$ transfection, H9C2 cells were treated with $5 \mathrm{ng}$ Notch1 recombinant protein in a hypoxic chamber (5\% $\mathrm{CO}_{2}$ and $95 \%$ nitrogen) for $2 \mathrm{~h}$.

Cell proliferation assay. To analyze cell proliferation, 2,000 cells/well were plated in 96-well plates and $10 \mu \mathrm{l}$ MTT was added to each well. The plates were then incubated for $4 \mathrm{~h}$ at $37^{\circ} \mathrm{C}$. The culture medium was removed, dimethyl sulfoxide was added to the wells and the plates were incubated for $20 \mathrm{~min}$ at $37^{\circ} \mathrm{C}$. A microplate reader was then used to measure absorbance of formazan at $492 \mathrm{nM}$.

Flow cytometric assay of apoptosis. To analyze apoptosis, H9C2 cells were transfected for $48 \mathrm{~h}$ and processed using the $10 \mu \mathrm{l}$ Annexin V-Fluorescein isothiocyanate/5 $\mu \mathrm{l}$ Propidium 


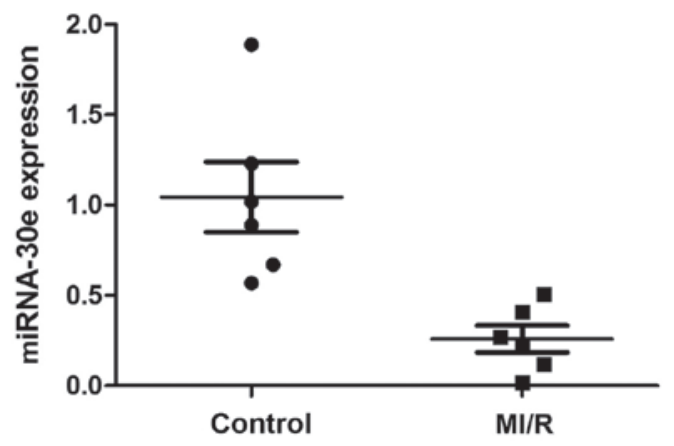

Figure 1. The expression of miRNA-30e was measured using reverse transcription-quantitative polymerase chain reaction and was lower in patients with MI/R compared with healthy controls. MI/R, myocardial ischemia/reperfusion injury; mi, micro.

Iodide Apoptosis Detection kit (BD Biosciences, San Jose, CA, USA) for $15 \mathrm{~min}$ in the dark. Analysis was performed using a BD FACSCanto ${ }^{\text {TM }}$ II (BD Biosciences) flow cytometer with FlowJo 7.6.1 software (FlowJo LLC, Ashland, OR, USA).

Analysis of caspase-3 activity. Prior to analysis of caspase-3 activity, cells were transfected for $48 \mathrm{~h}$ and lysed in radioimmunoprecipitation assay (RIPA) buffer (Beyotime Institute of Biotechnology, Haimen, China). Total protein was quantified using a BCA assay (Beyotime Institute of Biotechnology). Subsequently, $10 \mu \mathrm{g}$ protein was analyzed using a caspase-3 assay kit (cat. no., C1115; Beyotime Institute of Biotechnology) to determine caspase-3 activity. A microplate reader was used to measure absorbance at $405 \mathrm{nM}$.

Western blot analysis. Proteins were extracted from H9C2 cells following transfection for $48 \mathrm{~h}$ and subsequently lysed in RIPA buffer. Total protein was quantified using a BCA assay. A total of $50 \mu \mathrm{g}$ protein was separated using 6-12\% SDS-PAGE and transferred onto polyvinylidene difluoride membranes. Following blocking for $1 \mathrm{~h}$ with $5 \%$ bovine serum albumin (Beyotime Institute of Biotechnology) and TBS with $0.1 \%$ Tween-20 at $37^{\circ} \mathrm{C}$, membranes were incubated with primary antibodies against apoptosis regulator BAX (Bax), inducible nitric oxide synthase (iNOS, cat. no., sc-649; 1:500; Santa Cruz Biotechnology), microtubule-associated proteins 1A/1B light chain 3B (LC3, cat. no., 4599; 1:2,000), p62 (cat no., 88588; 1:2,000), Beclin-1 (cat. no., 3495; 1:2,000), neurogenic locus notch homolog protein 1 (Notch1, cat. no., 3608; 1:2,000), transcription factor Hes-1 (Hes1, cat. no., 11988; 1:2,000), phosphorylated (p)-Akt (cat. no., 4060; 1:2,000) and GAPDH (cat. no., 2118; 1:5,000; all from Cell Signaling Technology, Inc., Danvers, MA, USA) overnight at $4^{\circ} \mathrm{C}$. Membranes were subsequently incubated with anti-rabbit or anti-mouse AP-linked secondary antibody (cat. nos., 7054 and 7056, respectively; 1:5,000; Cell Signaling Technology Inc.) for $1 \mathrm{~h}$ at room temperature. Membranes were labeled with horseradish peroxidase and detected using the Pierce Enhanced Chemiluminescence kit (Thermo Fisher Scientific, Inc.) and results were analyzed using Image Lab software 3.0 (Bio-Rad Laboratories, Inc., Hercules, CA, USA).

ELISA assay. Following $48 \mathrm{~h}$ transfection, cells were lysed in RIPA buffer. Total protein was quantified using a BCA assay. A total of $10 \mu \mathrm{g}$ total protein was analyzed using ELISA kits against glutathione (GSH; cat. no., A006-2), GSH-peroxidase (PX; cat. no., A005) and superoxide dismutase (SOD; cat. no., A001-1-1). A microplate reader was used to measure absorbance at $450 \mathrm{nM}$.

Statistical analysis. All data are expressed as the mean \pm standard deviation. Two-way analysis of variance with Bonferroni multiple comparisons adjustments was used to assess the statistical significance of differences among groups. $\mathrm{P}<0.05$ was considered to indicate a statistically significant difference.

\section{Results}

MiRNA-30e expression is decreased in patients with MI/R. Changes in the expression of miRNA-30e were investigated in patients with $\mathrm{MI} / \mathrm{R}$ compared with a control group. It was demonstrated that miRNA-30e expression was markedly decreased in the serum of patients with MI/R compared with the control group (Fig. 1).

Si-miRNA-30e inhibits apoptosis. To investigate the effects of miRNA-30e on apoptosis, si-miRNA-30e was used to significantly inhibit miRNA-30e expression in $\mathrm{H} 9 \mathrm{C} 2$ cells $(\mathrm{P}<0.01$; Fig. 2A). The rate of cell proliferation was significantly increased in cells transfected with si-miRNA-30e compared with controls $(\mathrm{P}<0.01$; Fig. $2 \mathrm{~B})$. Furthermore, the rate of apoptosis was significantly decreased in cells treated with si-miRNA-30e compared with controls ( $\mathrm{P}<0.01$; Fig. 2C).

Si-miRNA-30e inhibits iNOS, Bax and caspase-3 expression. ELISA and western blot analysis was used to evaluate the effect of miRNA-30e on caspase-3 activity and the expression of iNOS and Bax in $\mathrm{H} 9 \mathrm{C} 2$ cells. Following inhibition of miRNA-30e by si-miRNA-30e, there was a significant
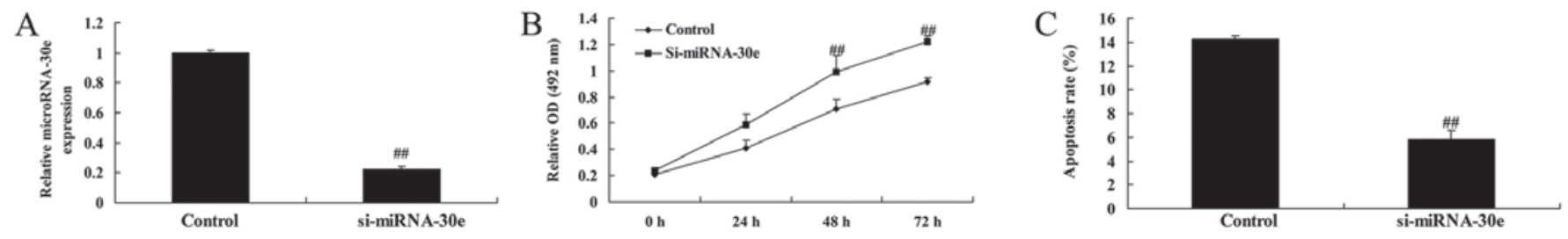

Figure 2. Si-miRNA-30e inhibits the apoptosis of H9C2 cells. (A) MiRNA-30e expression in H9C2 cells was measured following transfection with si-miRna-30e. The rate of (B) proliferation and (C) apoptosis was measured following transfection with si-miRNA-30e. ${ }^{\# \#} \mathrm{P}<0.01 \mathrm{vs}$. the control group. miRNA, microRNA; si, small interfering; OD, optical density. 
A
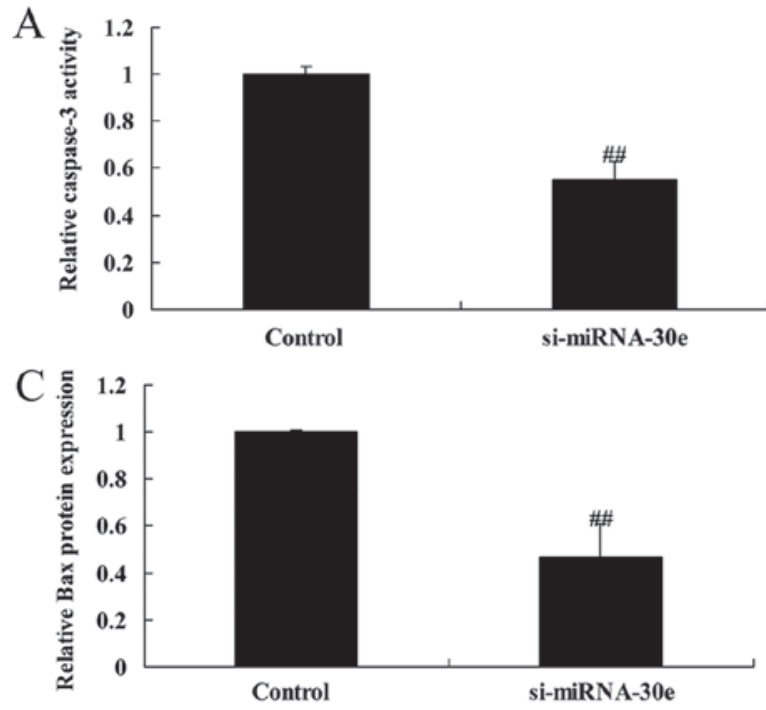

B

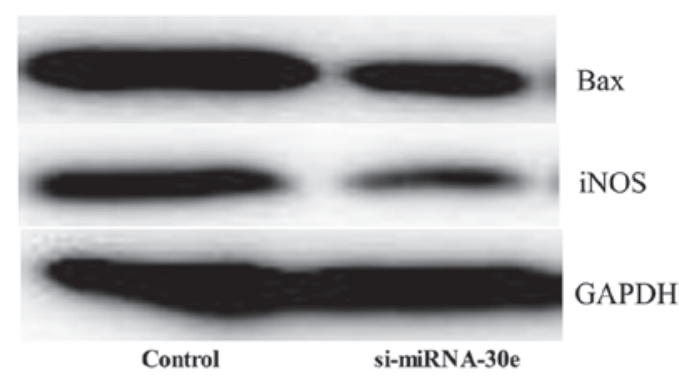

$\mathrm{D}$

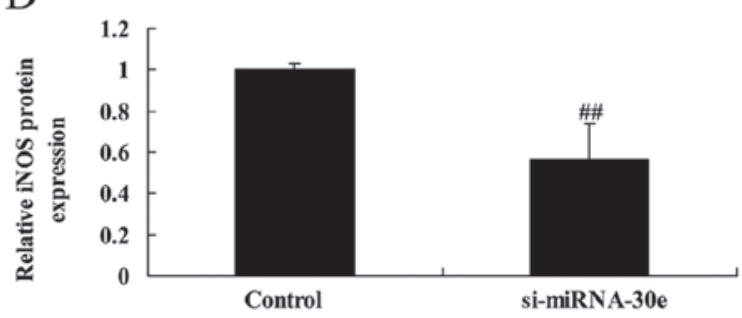

Figure 3. Si-miRNA-30e inhibits iNOS, Bax and caspase-3 expression in $\mathrm{H} 9 \mathrm{C} 2$ cells. (A) ELISA revealed that caspase-3 activity was significantly reduced in $\mathrm{H} 9 \mathrm{C} 2$ cells transfected with si-miRNA-30e. (B) Western blot analysis revealed a significant reduction in (C) Bax and (D) iNOS expression in H9C2 cells. ${ }^{\#} \mathrm{P}<0.01$ vs. the control group. miRNA, microRNA; si, small interfering; Bax, apoptosis regulator BAX; iNOS, inducible nitric oxide synthase.
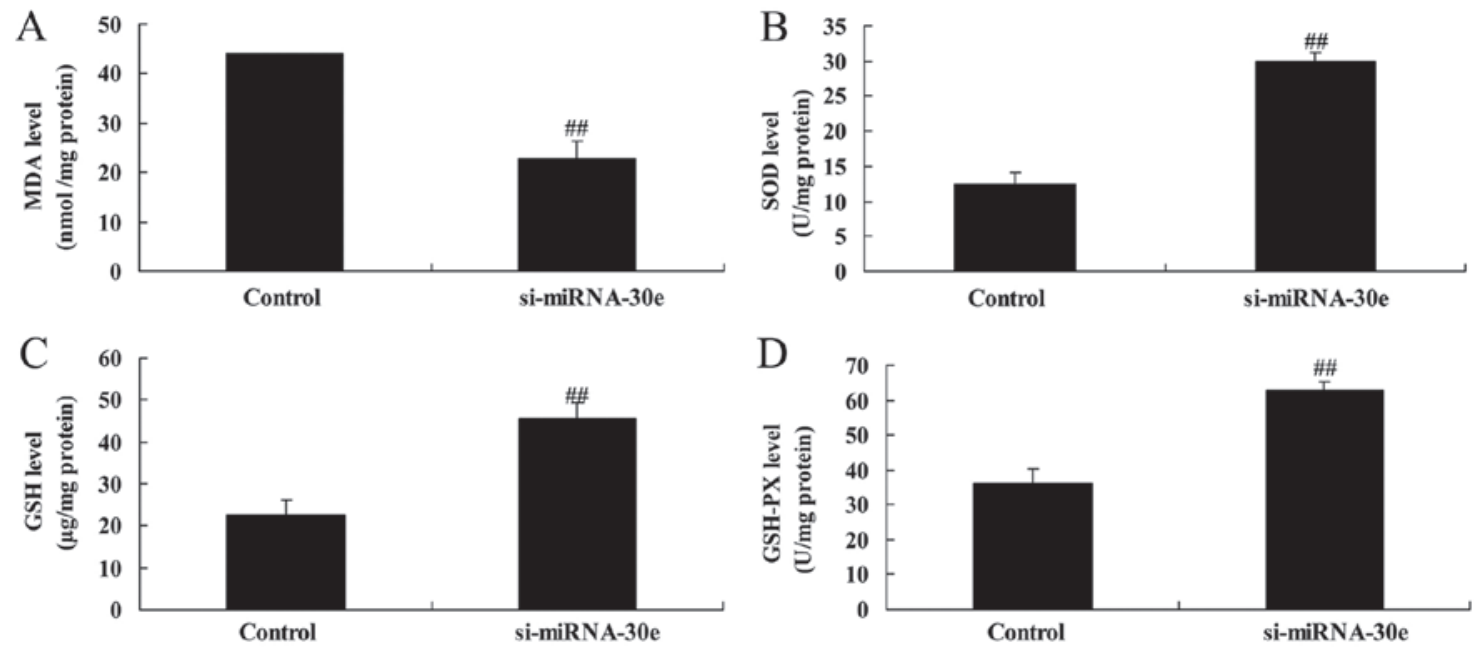

Figure 4. Knockdown of miRNA-30e increases the activity of proteins associated with oxidative stress in H9C2 cells. An ELISA kit was used to determine the activity of (A) MDA, (B) SOD, (C) GSH and (D) GSH-PX in H9C2 cells. ${ }^{\# /} \mathrm{P}<0.01$ vs. the control group. GSH, glutathione; PX, peroxides; SOD, superoxide dismutase; MDA, malondialdehyde; miRNA, micro RNA; si, small interfering.

reduction in caspase-3 activity compared with the control group $(\mathrm{P}<0.01$; Fig. 3A). Western blot analysis (Fig. 3B) revealed that the expression of Bax and iNOS were significantly reduced following inhibition of miRNA-30e $(\mathrm{P}<0.01$; Fig. 3C and D).

Knockdown of miRNA-30e increases the activity of proteins associated with oxidative stress. ELISA revealed that malondialdehyde activity was significantly decreased $(\mathrm{P}<0.01$; Fig. 4A) and that GSH, GSH-PX and SOD activities were significantly increased $(\mathrm{P}<0.01$; Fig. 4B-D) in $\mathrm{H} 9 \mathrm{C} 2$ cells following inhibition of miRNA-30e expression. These results indicate that miRNA-30e is able to regulate oxidative stress.

Silencing of miRNA-30e increases LC3, p62 and Beclin-1 expression. Western blot analysis was performed to determine whether miRNA-30e affects autophagy and the expression of autophagy-associated proteins in $\mathrm{H} 9 \mathrm{C} 2$ cells. The inhibition of miRNA-30e expression by si-miRNA-30e significantly increased the expression of LC3, p62 and Beclin-1 compared with the control group ( $\mathrm{P}<0.01$; Fig. 5). These are autophagy-associated proteins and these results suggest that a reduction of miRNA-30e expression, as observed in patients with MI/R, leads to an increase in cell autophagy.

miRNA-30e knockdown leads to a significant increase in Notch1, Hesl and p-Akt expression. The effect of si-miRNA-30e on Notch1, Hes1 and p-Akt signaling was examined in $\mathrm{H} 9 \mathrm{C} 2$ cells. Inhibition of miRNA-30e expression by si-miRNA-30e significantly increased the expression of Notch1, Hes1 and p-Akt compared with the control group $(\mathrm{P}<0.01$; Fig. 6). These results demonstrate that miRNA-30eprotects the heart against I/R injury via the Notch1/Hes1/Akt signaling pathway. 
A

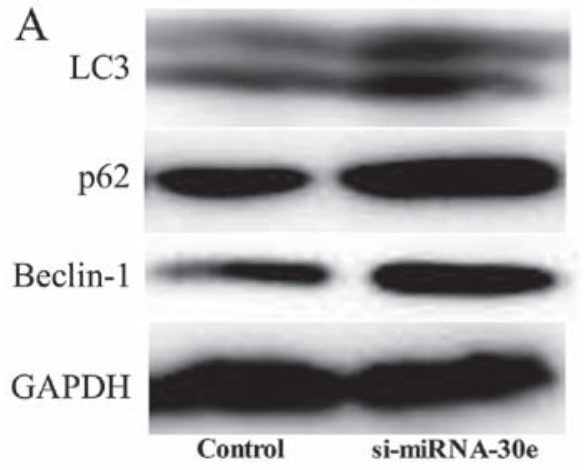

B

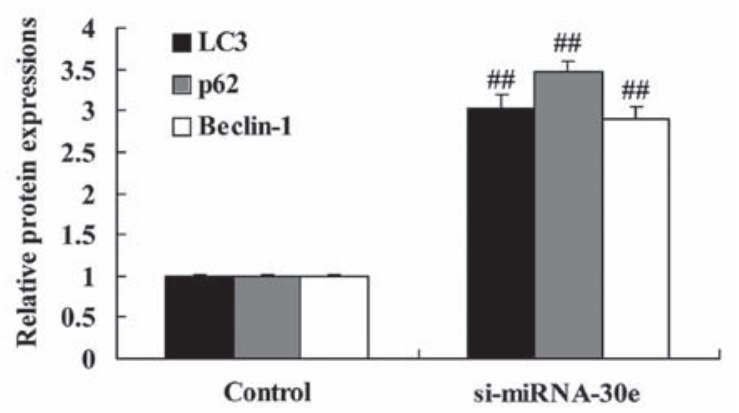

Figure 5. Inhibition of miRNA-30e significantly increased the expression of LC3, p62 and Beclin-1 in H9C2 cells. (A) Western blot analysis was performed and (B) the relative expression of LC3, p62 and Beclin-1 compared with the control group was determined. ${ }^{\# \#} \mathrm{P}<0.01$ vs. the control group. LC 3, microtubule-associated proteins 1A/1B light chain 3B; si, small interfering; miRNA, microRNA.
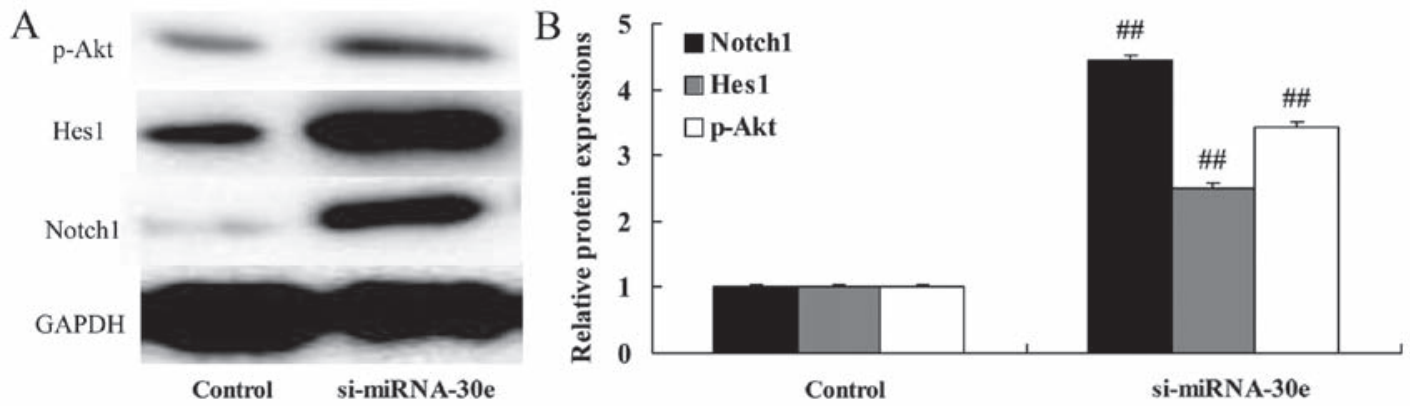

Figure 6. Treatment with si-miRNA-30e significantly increases Notch1, Hes1 and p-Akt expression in H9C2 cells. (A) The expression of Notch1, Hes1 and p-Akt was measured by western blot analysis and (B) and relative protein expression was determined compared with the control. ${ }^{\# \#} \mathrm{P}<0.01$ vs. the control group. Notch, neurogenic locus notch homolog protein; p, phosphorylated; Akt, protein kinase B; si, small interfering; miRNA, microRNA.
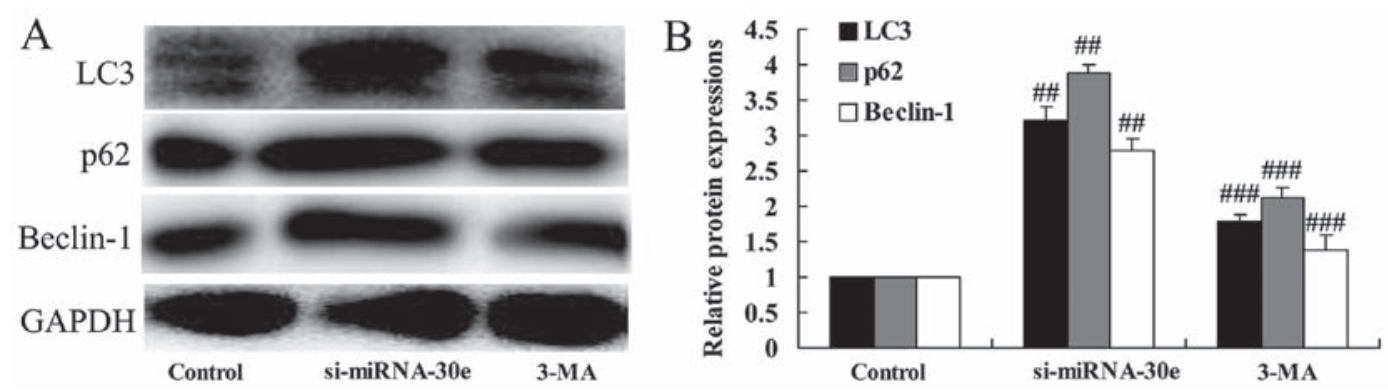

Figure 7. Inhibition of autophagy by 3-MA reverses the effect of si-miRNA-30e on LC3, p62 and Beclin-1 expression. (A) Western blot analysis was used to measure LC3, p62 and Beclin-1 expression following the treatment of $\mathrm{H} 9 \mathrm{C} 2$ cells with si-miRNA-30e and 3-MA. (B) Results were analyzed and compared with the control and the si-miRNA-30e groups. ${ }^{\# \#} \mathrm{P}<0.01$ vs. the control group, ${ }^{\# \# \#} \mathrm{P}<0.01$ vs. the si-miRNA-30e group. LC3, microtubule-associated proteins 1A/1B light chain 3B; si, small interfering; miRNA, microRNA; 3-MA, 3-methyladenine.

Inhibition of autophagy following treatment with 3-MA reverses the effect of si-miRNA-30e on LC3, p62 and Beclin-1 expression. To investigate whether inhibition of autophagy affects the function of si-miRNA-30e, H9C2 cells were treated with 3-MA. In cells treated with 3-MA, the expression of LC3, p62 and Beclin-1 were significantly reduced compared with the si-miRNA-30e group $(\mathrm{P}<0.01$; Fig. 7$)$. These results indicate that treatment with 3-MA is able to suppress autophagy and reduce the effects of si-miRNA-30e.

Autophagy inhibition reverses the effect of si-miRNA-30e on apoptosis. The proliferation and apoptosis of $\mathrm{H} 9 \mathrm{C} 2$ cells treated with either si-miRNA-30e or si-miRNA-30e and 3-MA were measured. Treatment with 3-MA reversed the effects of si-miRNA-30e on proliferation, as 3-MA-treated cells exhibited significantly lower proliferation rates compared with cells transfected with si-miRNA-30e $(\mathrm{P}<0.01$; Fig. 8A). In addition, 3-MA treated cells exhibited significantly higher rates of apoptosis compared with si-miRNA-30e cells, indicating that 3-MA reverses the effects of si-miRNA-30e on cellular apoptosis $(\mathrm{P}<0.01$; Fig. 8B).

Inhibition of autophagy following treatment with 3-MA reverses the effect of si-miRNA-30e on the expression of iNOS and Bax and caspase-3 activity. Inhibiting autophagy following treatment of $\mathrm{H} 9 \mathrm{C} 2$ cells with 3-MA significantly reversed the 

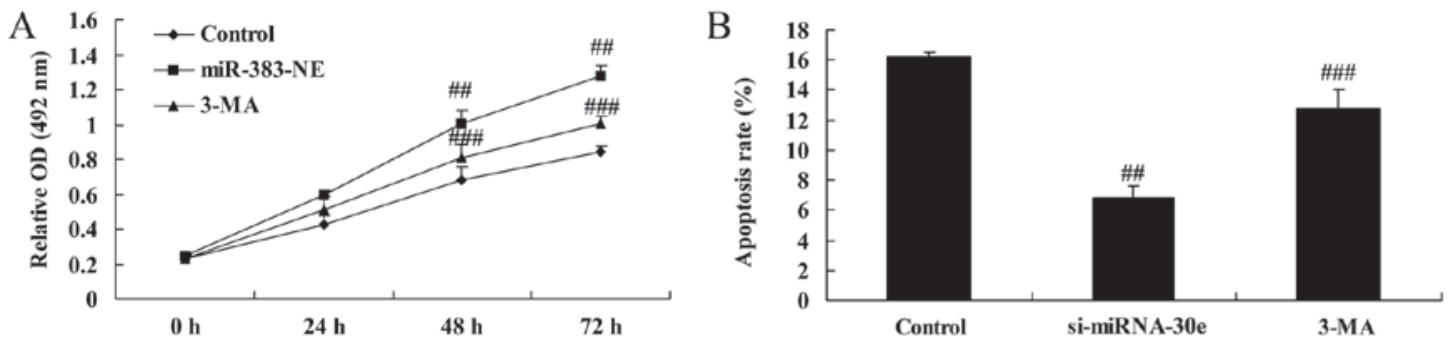

Figure 8. Inhibition of autophagy by 3-MA reverses the effect of si-miRNA-30e on apoptosis in H9C2 cells. (A) The proliferation (B) and apoptosis of H9C2 cells were measure in the control, mi-siRNA30e and 3-MA groups. ${ }^{\# \#} \mathrm{P}<0.01$ vs. the control group, ${ }^{\# \# \#} \mathrm{P}<0.01$ vs. the si-miRNA-30e group. si, small interfering; miRNA, microRNA; 3-MA, 3-methyladenine; OD, optical density.
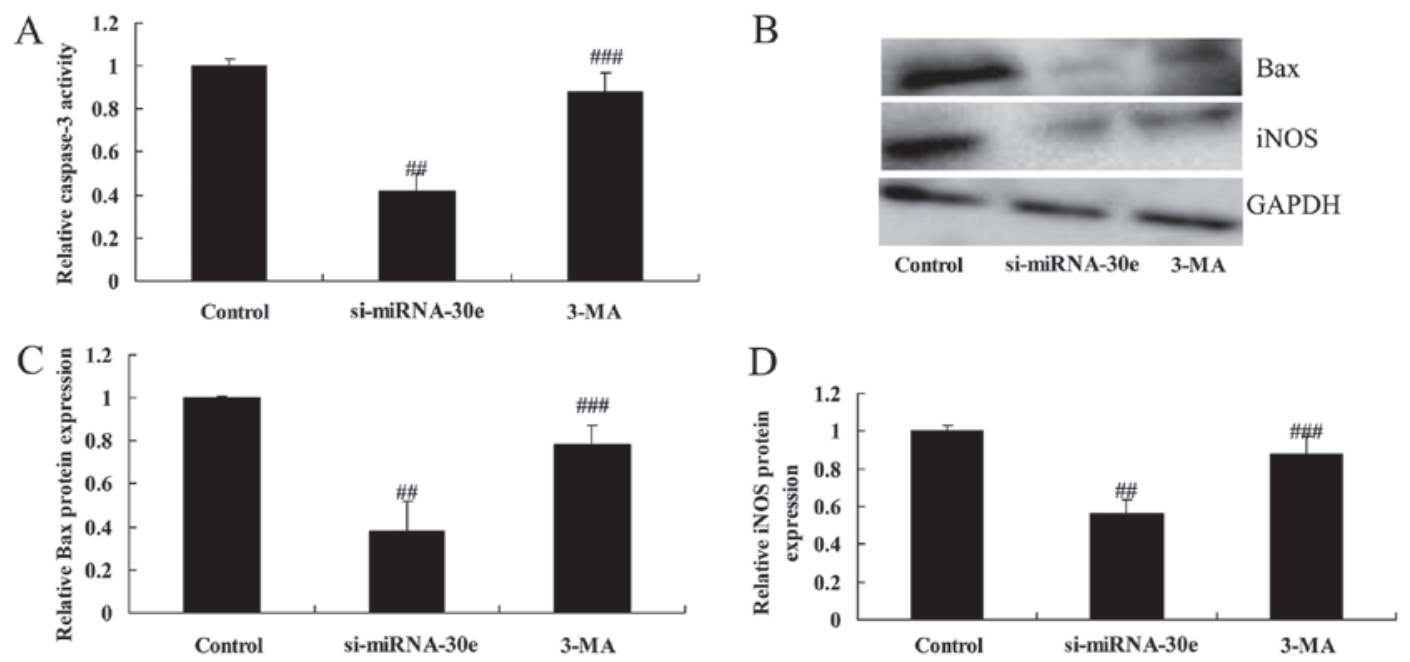

Figure 9. Inhibition of autophagy by 3-MA reverses the effect of si-miRNA-30e on iNOS, Bax and caspase-3. (A) Caspase-3 activity was measured by

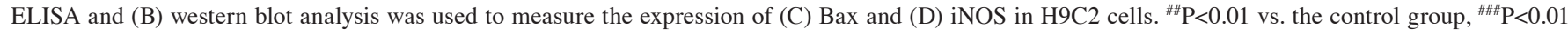
vs. the si-miRNA-30e group. Bax, apoptosis regulator BAX; iNOS, inducible nitric oxide synthase; 3-MA, 3-methyladenine; si, small interfering; miRNA, microRNA.
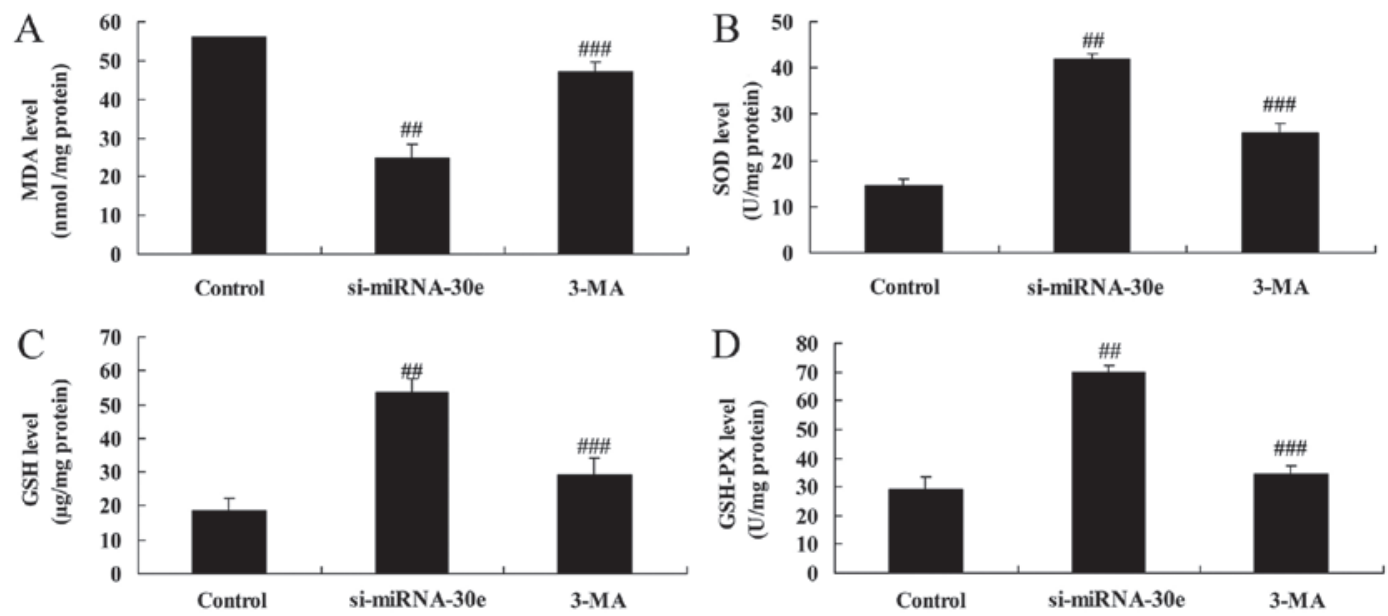

Figure 10. Inhibition of autophagy by 3-MA reverses the effect of si-miRNA-30e on oxidative stress. ELISA was used to measure levels of (A) MDA (B), SOD (C), GSH and (D) GSH-PX. ${ }^{\# \#} \mathrm{P}<0.01$ vs. the control group, ${ }^{\# \# \#} \mathrm{P}<0.01$ vs. the si-miRNA-30e group. 3-MA, 3-methyladenine; GSH, glutathione; PX, peroxides; SOD, superoxide dismutase; MDA, malondialdehyde; si, small interfering; miRNA, microRNA.

inhibition of caspase-3 activity, as well as the inhibition of Bax and iNOS expression by si-miRNA-30e $(\mathrm{P}<0.01 ;$ Fig. 9).

Treatment with 3-MA reverses the effect of si-miRNA-30e on oxidative stress. The effect of autophagy on the effect of
si-miRNA-30e on the expression of proteins in $\mathrm{H} 9 \mathrm{C} 2$ cells associated with oxidative stress was investigated (Fig. 10). Inhibition of autophagy following treatment with 3-MA significantly reduced the increase of SOD, GSH and GSH-PX activity compared with the si-miRNA-302 group ( $\mathrm{P}<0.01$; Fig. 10B-D 

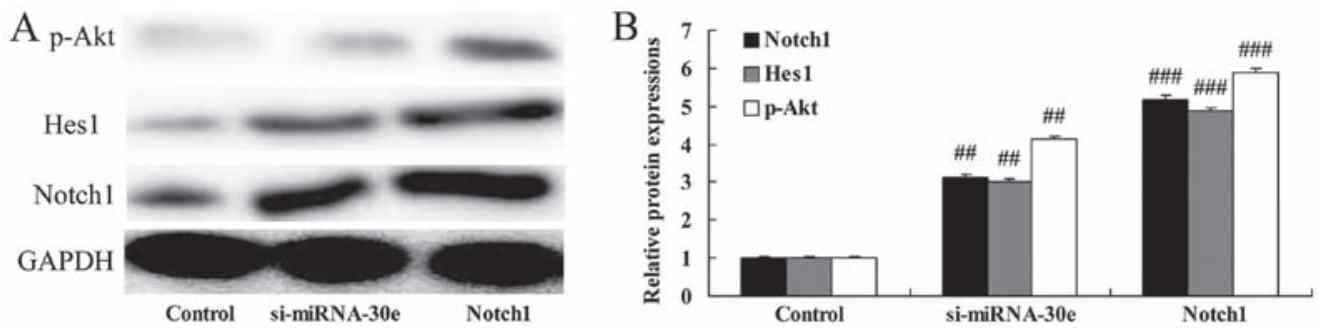

Figure 11. Notch1 promotion increases the effect of si-miRNA-30e on Notch1, Hes1 and p-Akt expression. (A) Western blot analysis was used to measure the expression of Notch1, Hes1 and p-Akt in $\mathrm{H} 9 \mathrm{C} 2$ cells. (B) The results were then quantified. ${ }^{\# \#} \mathrm{P}<0.01$ vs. the control group, ${ }^{\# \# ~} \mathrm{P}<0.01$ vs. the si-miRNA-30e group. Notch, neurogenic locus notch homolog protein; si, small interfering; miRNA, microRNA; p-, phosphorylated; Akt, protein kinase B.
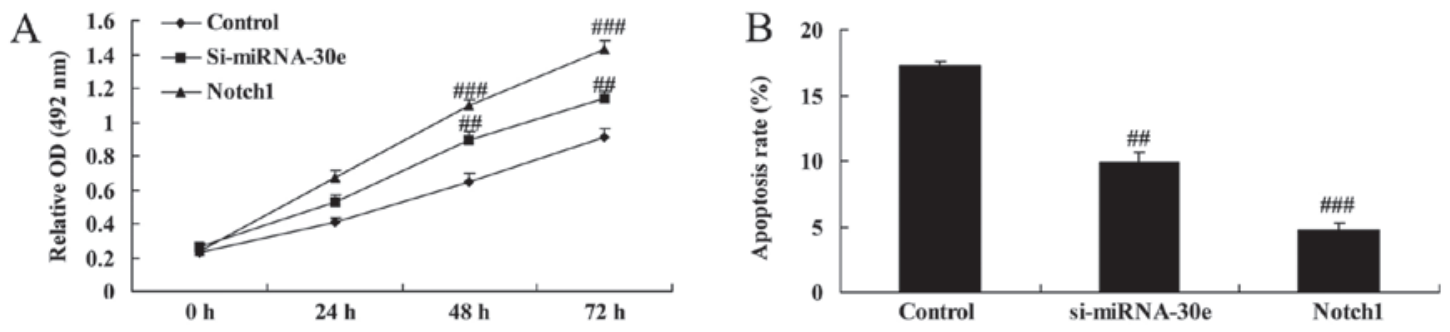

Figure 12. Notch1 promotion enhances the effect of si-miRNA-30e on apoptosis in $\mathrm{H} 9 \mathrm{C} 2$ cells. (A) The proliferation and (B) apoptosis rates of H9C2 cells was measured in the control, mi-siRNA30e and Notch1 groups. ${ }^{\# \#} \mathrm{P}<0.01$ vs. the control group, ${ }^{\# \#} \mathrm{P}<0.01$ vs. the si-miRNA-30e group. Notch, neurogenic locus notch homolog protein; si, small interfering; miRNA, microRNA; OD, optical density.
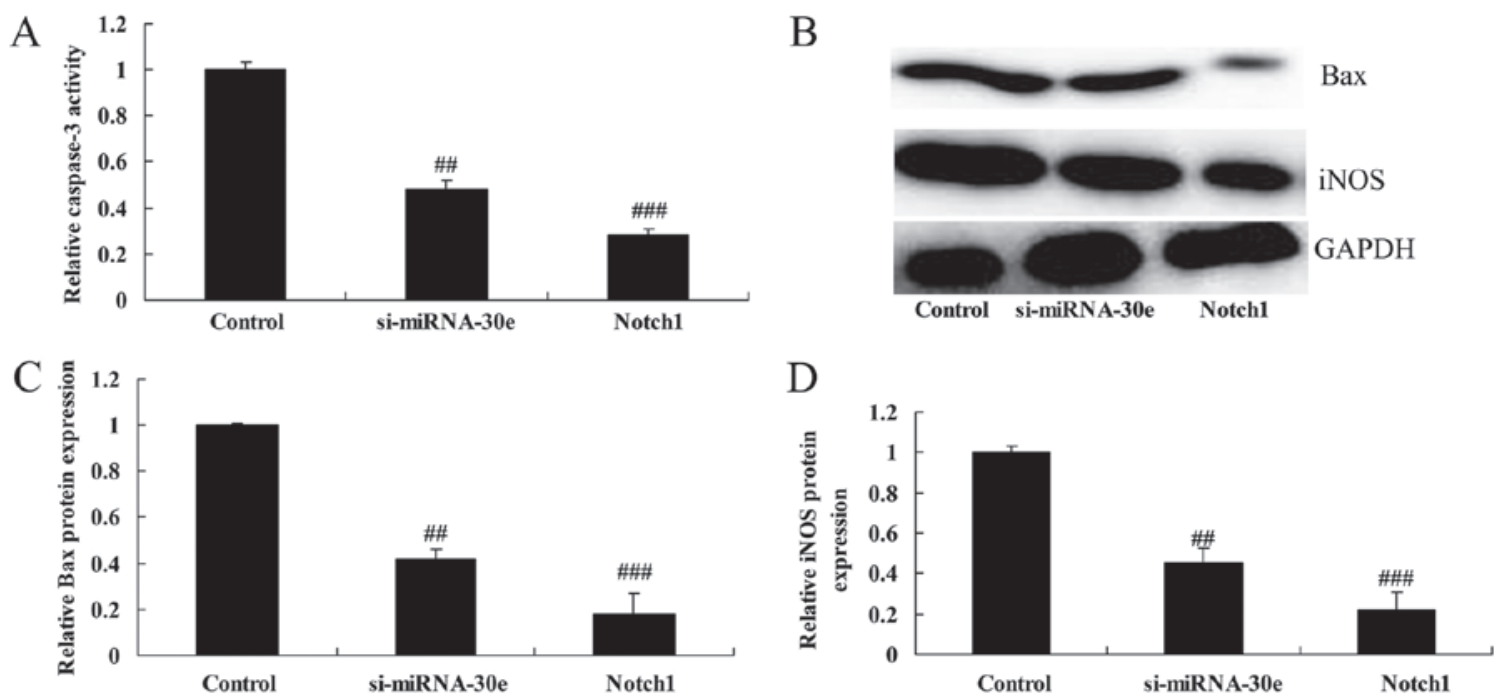

Figure 13. Notch1 promotion increases the effect of si-miRNA-30e on the expression of iNOS and Bax and caspase-3 activity. (A) ELISA was used to measure caspase-3 activity and (B) western blot analysis was used to measure the expression of (C) Bax and (D) iNOS in H9C2 cells. ${ }^{\# \prime} \mathrm{P}<0.01$ vs. the control group,

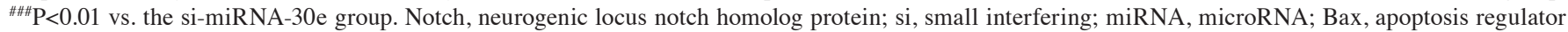
BAX; iNOS, inducible nitric oxide synthase.

respectively). Furthermore, treatment with 3-MA significantly reversed the decrease in MDA activity that occurred following treatment with si-miRNA-30e alone $(\mathrm{P}<0.01$; Fig. 10A).

Notchl promotion enhances the effect of si-miRNA-30e on Notch1, Hes1 and p-Akt expression. The effect of Notch1 expression on Notch1, Hes1 and p-Akt expression was investigated. The Notch1 recombinant protein was administered to $\mathrm{H} 9 \mathrm{C} 2$ cells as well as si-miRNA-30e and the effect was compared to that of $\mathrm{H} 9 \mathrm{C} 2$ cells treated with si-miRNA-30e alone. The Notch1 recombinant protein significantly enhanced the effect of si-miRNA-30e on Notch1, Hes1 and p-Akt expression compared with treatment with si-miRNA-30e alone $(\mathrm{P}<0.01$; Fig. 11).

Notch1 promotion increases the effect of si-miRNA-30e on the apoptosis and proliferation of cells. It was determined whether Notch1 affects the function of si-miRNA-30e on the apoptosis and proliferation of $\mathrm{H} 9 \mathrm{C} 2$ cells. Compared with the si-miRNA-30e group, the promotion of Notch1 expression significantly increased proliferation $(\mathrm{P}<0.01$; Fig. 12A) and significantly decreased apoptosis $(\mathrm{P}<0.01$; Fig. 12B). 

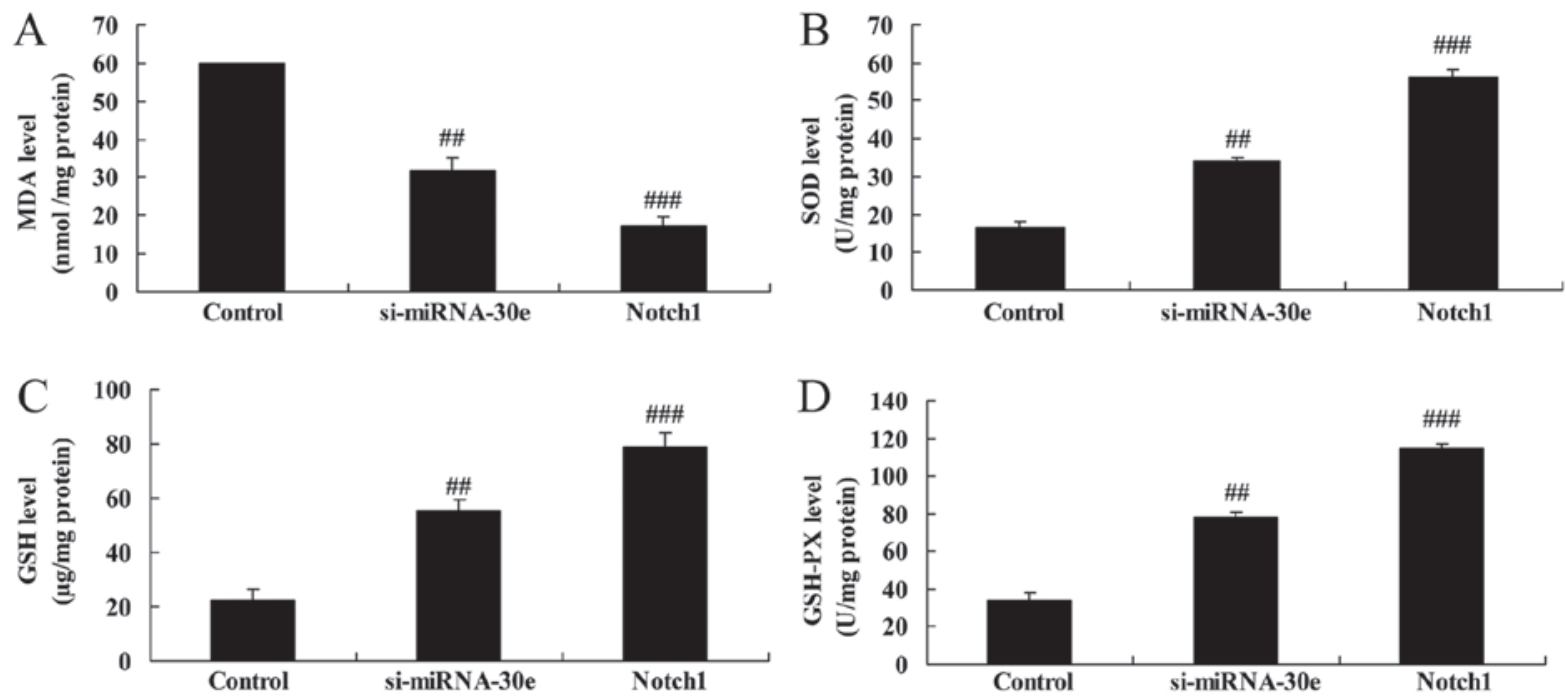

Figure 14. Notch1 promotion increases the effect of si-miRNA-30e on oxidative stress. ELISA was used to measure the activity of (A) MDA (B), SOD (C), GSH and (D) GSH-PX. ${ }^{\# \#} \mathrm{P}<0.01$ vs. the control group, ${ }^{\# \#} \mathrm{P}<0.01$ vs. the si-miRNA-30e group. Notch, neurogenic locus notch homolog protein; GSH, glutathione; PX, peroxides; SOD, superoxide dismutase; MDA, malondialdehyde; si, small interfering; miRNA, microRNA.

Notch1 promotion enhances the effect of si-miRNA-30e on $i N O S$ and Bax expression and caspase-3 activity. The promotion of Notch1 expression significantly increased the effect of si-miRNA-30e on the inhibition of caspase-3 activity, as caspase-3 activity was significantly lower in $\mathrm{H} 9 \mathrm{C} 2$ cells treated with Notch1 recombinant protein compared with si-miRNA-30e treated cells $(\mathrm{P}<0.01$; Fig. 13A). Furthermore, western blot analysis revealed that treatment with Notch1 recombinant protein led to a significant reduction in the expression of Bax and iNOS compared with the si-miRNA-30e group $(\mathrm{P}<0.01$; Fig. 13B-D).

Notch1 promotion increases the effect of si-miRNA-30e on the activity of proteins associated with oxidative stress. The effect of Notch1 on the function of si-miRNA-30e on oxidative stress was investigated. MDA activity was significantly lower in cells treated with Notch1 recombinant protein compared with si-miRNA-30e-treated cells $(\mathrm{P}<0.01$; Fig. 14A). However, the activity of GSH, GSH-PX and SOD were all significantly increased in cells treated with Notch1 recombinant protein compared with si-miRNA-30e-treated cells $(\mathrm{P}<0.01$; Fig. 14B-D).

\section{Discussion}

$\mathrm{MI} / \mathrm{R}$ is a condition that severely affects human health. Due to the development of novel anticoagulants and antiplatelet agents, as well as the continuous development of intervention catheter technology and coronary artery bypass grafting, the survival rate of patients with MI/R has improved markedly since 2012 (22). However, myocardial cells lack the capacity to regenerate, which results in an irreversible loss of myocardial cells following necrosis. This reduces cardiac function and may progress to heart failure, which has a high long-term mortality rate (23). Promoting repair of the MI/R region is key to preventing post-MI/R heart failure and lowering long-term mortality rates (23). The present study revealed that
miRNA-30e expression was decreased in patients with MI/R. Furthermore, it was demonstrated that miRNA-30e suppression significantly inhibited apoptosis and significantly reduced Bax protein expression and caspase- 3 activity in $\mathrm{H} 9 \mathrm{C} 2$ cells.

Studies have indicated that autophagy is involved in the process of myocardial remodeling. It has been demonstrated that treatment with the autophagy inducer sirolimus delays the process of ventricular remodeling (10). In MI/R, autophagy may increase intracellular energy supplies and produce sufficient energy for cell survival; it may therefore be considered a protective process (8). Zeng et al (24) reported that autophagy was enhanced in animal models of MI/R. Autophagy was activated in the sub-acute and chronic phases of MI/R and the expression of the autophagosome markers LC3-II and p62, as well as the expression of cathepsin, were upregulated (24). During reperfusion, a large number of myocardial cells die, which may be caused by Beclin-1-induced autophagy (25). In the present study, suppression of miRNA-30e increased LC3, p62 and Beclin-1 expression in H9C2 cells. Lai et al (19) demonstrated that miRNA-30e exhibited cardioprotection in rats with doxorubicin-induced heart failure via autophagy.

During the embryonic cardiac developmental phase, Notch1 is highly expressed in the immature myocardium but its expression decreases markedly following birth (13). However, the expression of Notch signaling pathway-related molecules is increased in the adult myocardium following $\mathrm{MI} / \mathrm{R}$, indicating that the Notch signaling pathway is closely associated with heart injury (12). It has been suggested that the activated Notch signal pathway restricts the myocardial ischemic size and improves myocardial function following MI/R (13). Therefore, the increased expression of Notch signaling pathway-associated molecules in the myocardial cells of adults following MI/R in the adult myocardium may be a protective mechanism (26). The results of the present study revealed that suppression of miRNA-30e-induced Notch1 expression and promotion of Notch1 in $\mathrm{H} 9 \mathrm{C} 2$ cells significantly increased the effect of si-miRNA-30e on cell 
apoptosis, Bax and iNOS expression and caspase-3 activity in H9C2 cells.

Notch1 intracellular cytoplasmic domain (NICD) is translocated into the cell nucleus and regulates Hes1 gene expression by associating with ubiquitous transcription factors and centromere-binding protein 1 (17). It has been demonstrated that the Notch signaling pathway serves an important role in the onset and development of the cardiovascular system, as well as during pathophysiological processes (17). The roles of the Notch1 receptor and its downstream signal molecule Hes1 in ischemic heart disease have been investigated (27). Activation of the Notch1 molecule during MI/R may activate the downstream Hes1 molecule and thus inhibit the expression of phosphatase and tensin homolog, thereby alleviating MI/R injury (17). Activation of the myocardial Notch1 signaling pathway contributes to the alleviation of MI/R injury-induced oxidative stress and nitrative stress injury by activating the downstream Hes1 signal, thus protecting the ischemic myocardium (17). The Akt signaling pathway serves a crucial role in processes including cell survival, growth and migration; it may also serve as a survival signal during myocardial ischemia and activation of this signal exerts anti-apoptotic effects via up- and downstream signaling $(16,17)$. The results of the present study demonstrated that suppression of miRNA-30e increased Hesl and p-Akt expression. Shan et al (28) revealed that miRNA-30e regulates the abnormal differentiation of small intestinal epithelial cells via the $\delta$-like canonical notch ligand 4/NICD/Hes1 signaling pathway.

Preventing the obstruction of blood flow in patients with $\mathrm{MI} / \mathrm{R}$ is a problem that occurs during coronary artery reperfusion therapy and requires solving (29). The pathogenesis of blood flow obstruction is complicated; previous studies have reported that it occurs due to the combined action of multiple factors, including ischemic microvascular injury in the infarct area, release of oxygen radicals, endothelial injury, inflammatory response, platelet activation and microthrombosis $(29,30)$. In the present study, it was revealed that suppression of miRNA-30e in H9C2 cells decreased oxidative stress.

Increased iNOS expression and the resulting excessive production of $\mathrm{NO}$ causes of cell death following MI/R injury (31). NOS catalyzes arginine to produce citrulline and release NO (31). Minimal NO is produced under normal physiological conditions and under these conditions, it inhibits neutrophil adhesion and platelet aggregation, dilates blood vessels, regulates vascular tone and blood flow volume and has protective effects on heart function (32). However, activation of iNOS during MI/R causes excessive NO production, which may cause the injury or death of nerve cells (32). In the present study, suppression of miRNA-30e decreased iNOS expression and the inhibition of autophagy following treatment with 3-MA reversed the effects of si-miRNA-30e on apoptosis, Bax and iNOS expression, caspase-3 activity and oxidative stress.

In conclusion, the results of the present study indicate that miRNA-30e serves a significant role in suppressing $\mathrm{MI} / \mathrm{R}$-induced apoptosis and oxidative stress via autophagy and the Notch1/Hes1/Akt signaling pathway. Further studies are required to determine the clinical significance of these effects and to develop novel therapies for patients with $M I / R$, as treating this disease remains clinically challenging.

\section{Acknowledgements}

Not applicable.

\section{Funding}

The present study was supported by the Science and Technology Project of Shaanxi Province (grant no. 2007K13-03).

\section{Availability of data and materials}

The analyzed data sets generated during the study are available from the corresponding author on reasonable request.

\section{Authors' contributions}

All authors have seen the manuscript and approved submission to your journal. JZ designed the experiment; JL, BK, QY and TS performed the experiment; JZ and JL analyzed the data; JZ wrote the manuscript.

\section{Ethics approval and consent to participate}

The present study was approved by the Ethics Committee of the First Affiliated Hospital of Xi'an Jiaotong University (Xi'an, China). All patients provided written informed consent prior to the collection of blood samples.

\section{Consent for publication}

Not applicable.

\section{Competing interests}

The authors declare that they have no competing interests.

\section{References}

1. Gu YL, Kampinga MA, Wieringa WG, Fokkema ML, Nijsten MW, Hillege HL, van den Heuvel AF, Tan ES, Pundziute $\mathrm{G}$, van der Werf $\mathrm{R}$, et al: Intracoronary versus intravenous administration of abciximab in patients with ST-segment elevation myocardial infarction undergoing primary percutaneous coronary intervention with thrombus aspiration: The comparison of intracoronary versus intravenous abciximab administration during emergency reperfusion of ST-segment elevation myocardial infarction (CICERO) trial. Circulation 122: 2709-2717, 2010.

2. Garcia-Dorado D, García-del-Blanco B, Otaegui I, Rodríguez-Palomares J, Pineda V, Gimeno F, Ruiz-Salmerón R, Elizaga J, Evangelista A, Fernandez-Avilés F, et al: Intracoronary injection of adenosine before reperfusion in patients with ST-segment elevation myocardial infarction: A randomized controlled clinical trial. Int J Cardiol 177: 935-941, 2014.

3. Hausenloy D, Kunst G, Boston-Griffiths E, Kolvekar S, Chaubey S, John L, Desai J and Yellon D: The effect of cyclosporin-A on peri-operative myocardial injury in adult patients undergoing coronary artery bypass graft surgery: A randomised controlled clinical trial. Heart 100: 544-549, 2014.

4. Liang Y, Li YP, He F, Liu XQ and Zhang JY: Long-term, regular remote ischemic preconditioning improves endothelial function in patients with coronary heart disease. Braz J Med Biol Res 48: 568-576, 2015.

5. Ma N, Bai J, Zhang W, Luo H, Zhang X, Liu D and Qiao C: Trimetazidine protects against cardiac ischemia/reperfusion injury via effects on cardiac miRNA-21 expression, Akt and the Bcl-2/Bax pathway. Mol Med Rep 14: 4216-4222, 2016. 
6. Wang Y, Ouyang M, Wang Q and Jian Z: MicroRNA-142-3p inhibits hypoxia/reoxygenation-induced apoptosis and fibrosis of cardiomyocytes by targeting high mobility group box 1 . Int J Mol Med 38: 1377-1386, 2016.

7. Hendgen-Cotta UB, Messiha D, Esfeld S, Deenen R, Rassaf T and Totzeck M: Inorganic nitrite modulates miRNA signatures in acute myocardial in vivo ischemia/reperfusion. Free Radic Res 51: 91-102, 2017.

8. Wang ZG, Wang Y, Huang Y, Lu Q, Zheng L, Hu D, Feng WK, Liu YL, Ji KT, Zhang HY, et al: bFGF regulates autophagy and ubiquitinated protein accumulation induced by myocardial ischemia/reperfusion via the activation of the PI3K/Akt/mTOR pathway. Sci Rep 5: 9287, 2015.

9. Duan Q, Yang W, Jiang D, Tao K, Dong A and Cheng H: Spermine ameliorates ischemia/reperfusion injury in cardiomyocytes via regulation of autophagy. Am J Transl Res 8: 3976-3985, 2016.

10. Song H, Yan C, Tian X, Zhu N, Li Y, Liu D, Liu Y, Liu M, Peng C, Zhang $\mathrm{Q}$, et al: CREG protects from myocardial ischemia/reperfusion injury by regulating myocardial autophagy and apoptosis. Biochim Biophys Acta 1863: 1893-1903, 2017.

11. Pei H, Song X, Peng C, Tan Y,Li Y, Li X, Ma S, Wang Q, Huang R, Yang D, et al: TNF- $\alpha$ inhibitor protects against myocardial ischemia/reperfusion injury via Notch1-mediated suppression of oxidative/nitrative stress. Free Radic Biol Med 82: 114-121, 2015.

12. Pei H, Yu Q, Xue Q, Guo Y, Sun L, Hong Z, Han H, Gao E, $\mathrm{Qu}$ Y and Tao L: Notch1 cardioprotection in myocardial ischemia/reperfusion involves reduction of oxidative/nitrative stress. Basic Res Cardiol 108: 373, 2013.

13. Zhou XL, Wan L and Liu JC: Activated Notch1 reduces myocardial ischemia reperfusion injury in vitro during ischemic postconditioning by crosstalk with the RISK signaling pathway. Chin Med J 126: 4545-4551, 2013.

14. Boccalini G, Sassoli C, Formigli L, Bani D and Nistri S: Relaxin protects cardiac muscle cells from hypoxia/reoxygenation injury: Involvement of the Notch-1 pathway. FASEB J 29: 239-249, 2015

15. Zhou XL, Zhao Y, Fang YH, Xu QR and Liu JC: Hes1 is upregulated by ischemic postconditioning and contributes to cardioprotection. Cell Biochem Funct 32: 730-736, 2014.

16. Yu L, Li F, Zhao G, Yang Y, Jin Z, Zhai M, Yu W, Zhao L, Chen W, Duan W and Yu S: Protective effect of berberine against myocardial ischemia reperfusion injury: Role of Notch1/Hes1-PTEN/Akt signaling. Apoptosis 20: 796-810, 2015.

17. Yu L, Liang H, Lu Z, Zhao G, Zhai M, Yang Y, Yang J, Yi D, Chen W, Wang X, et al: Membrane receptor-dependent Notch1/Hes1 activation by melatonin protects against myocardial ischemia-reperfusion injury: In vivo and in vitro studies. J Pineal Res 59: 420-433, 2015.

18. Lavie L: Oxidative stress in obstructive sleep apnea and intermittent hypoxia-revisited-the bad ugly and good: Implications to the heart and brain. Sleep Med Rev 20: 27-45, 2015.

19. Lai L, Chen J, Wang N, Zhu G, Duan $X$ and Ling $F$ : MiRNA-30e mediated cardioprotection of ACE2 in rats with Doxorubicin-induced heart failure through inhibiting cardiomyocytes autophagy. Life Sci 169: 69-75, 2017.

20. Livak KJ and Schmittgen TD: Analysis of relative gene expression data using real-time quantitative PCR and the $2^{-\Delta C_{\mathrm{T}}}$ method. Methods 25: 402-408, 2001.
21. Xue R, Lei S, Xia ZY, Wu Y, Meng Q, Zhan L, Su W, Liu H, Xu J, Liu Z, et al: Selective inhibition of PTEN preserves ischaemic post-conditioning cardioprotection in STZ-induced Type 1 diabetic rats: Role of the PI3K/Akt and JAK2/STAT3 pathways. Clin Sci 130: 377-392, 2016.

22. Liu Z, Zhao L, Hong D and Gao J: Remote ischaemic preconditioning reduces myocardial ischaemic reperfusion injury in patients with ST-elevation myocardial infarction undergoing primary percutaneous coronary intervention. Acta Cardiol 71: 596-603, 2016.

23. Pöss J, Desch S, Eitel C, de Waha S, Thiele H and Eitel I: Left ventricular thrombus formation after ST-segment-elevation myocardial infarction: Insights from a cardiac magnetic resonance multicenter study. Circ Cardiovasc Imaging 8: e003417, 2015.

24. Zeng C, Li H, Fan Z, Zhong L, Guo Z, Guo Y and Xi Y: Crocin-elicited autophagy rescues myocardial ischemia/reperfusion injury via paradoxical mechanisms. Am J Chin Med 44: 515-530, 2016.

25. Thapalia BA, Zhou Z and Lin X: Autophagy, a process within reperfusion injury: An update. Int J Clin Exp Pathol 7: 8322-8341, 2014.

26. Zhou XL, Wan L, Xu QR, Zhao Y and Liu JC: Notch signaling activation contributes to cardioprotection provided by ischemic preconditioning and postconditioning. J Transl Med 11: 251, 2013.

27. Zhang S, Wang P, Ren L, Hu C and Bi J: Protective effect of melatonin on soluble $\mathrm{A} \beta_{1-42}$-induced memory impairment, astrogliosis, and synaptic dysfunction via the Musashi1/Notch1/ Hes1 signaling pathway in the rat hippocampus. Alzheimers Res Ther 8: 40, 2016.

28. Shan TD, Ouyang H, Yu T, Li JY, Huang CZ, Yang HS, Zhong W, Xia ZS and Chen QK: miRNA-30e regulates abnormal differentiation of small intestinal epithelial cells in diabetic mice by downregulating Dl14 expression. Cell Prolif 49: 102-114, 2016.

29. Zhou Q, Cao J and Chen L: Apelin/APJ system: A novel therapeutic target for oxidative stress-related inflammatory diseases (Review). Int J Mol Med 37: 1159-1169, 2016

30. Bagheri F, Khori V, Alizadeh AM, Khalighfard S, Khodayari S and Khodayari H: Reactive oxygen species-mediated cardiac-reperfusion injury: Mechanisms and therapies. Life Sci 165: 43-55, 2016.

31. Wang G, Li X, Wang H, Wang Y, Zhang L, Zhang L, Liu B and Zhang M: Later phase cardioprotection of ischemic post-conditioning against ischemia/reperfusion injury depends on iNOS and PI3K-Akt pathway. Am J Transl Res 7: 2603-2611, 2015.

32. Sun N, Wang H and Wang L: Protective effects of ghrelin against oxidative stress, inducible nitric oxide synthase and inflammation in a mouse model of myocardial ischemia/reperfusion injury via the HMGB1 and TLR4/NF- $\kappa \mathrm{B}$ pathway. Mol Med Rep 14: 2764-2770, 2016.

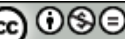

This work is licensed under a Creative Commons Attribution-NonCommercial-NoDerivatives 4.0 International (CC BY-NC-ND 4.0) License. 